\title{
Phase and Time Variations in VLF Propagation Over Long Distances
}

\author{
D. D. Crombie
}

\section{Contribution From the Central Radio Propagation Laboratory, National Bureau of Standards, Boulder, Colo.}

(Received June 9, 1964)

\begin{abstract}
It is argued that the custom of referring to changes in the phase of VLF signals as changes in transmission time is incorrect, is likely to lead to confusion and should be abandoned.
\end{abstract}

In recent vears various organizations have made observations of the phase frequency stabilized VLF signals relative to local frequency standards. These observations show that the phase of the received signal exhibits diurnal changes. Moreover, phase changes occur during solar flares and other geophysical disturbances.

It has proved useful for some purposes [Pierce, 1957 and Blackband, 1964 for example] to express these phase changes $(\Delta \phi)$ in terms of equivalent time changes $\left(\Delta T_{\phi}\right)$ by means of the relationship

$$
\Delta T_{\phi}=P \Delta \phi / 2 \pi=\frac{\Delta \phi}{2 \pi f}=\frac{\Delta \phi}{2 \pi} \frac{\lambda}{v}
$$

where $P, f, v, \lambda$ are the period, frequency, free space velocity of propagation, free space wavelength, and $\Delta \phi$ is measured in radians. The phase change $\Delta T_{\phi}$ is expressed in units of time and is then often interpreted as a change of "transmission time." The validity of this interpretation will be discussed here.

Transmission time is usually defined [Brillouin, 1960] (in a medium which does not exhibit anomalous dispersion) as the time which elapses between the transmission and reception at a point remote from the transmitter of a recognizable signal. Thus the transmission time is given by the propagation distance divided by the group velocity. Only when the group and phase velocities are equal is it valid to interpret a change in phase delay as a change in transmission time.

The transmission of VLF signals over long distances can be considered correctly from either the viewpoint of ray theory or of waveguide theory. For the sake of illustration only, the simplest possible model is one in which the earth and ionosphere are flat, have infinite electrical conductivity, and are separated by a distance $h$. Using a single ray treatment, however, it is easy to show that the propagation phase delay $\phi$ for a distance $2 d$, measured on the ground, for a one hop ray, and assuming $h<<d$, is given by

$$
\phi=\frac{4 \pi f}{c} d\left(1+\frac{1}{2} \frac{h^{2}}{d^{2}}\right)
$$

Equation (2) can be written, using (1), as

$$
T_{\phi}=\frac{\phi}{2 \pi f}=\frac{2 d}{c}\left(1+\frac{1}{2} \frac{h^{2}}{d^{2}}\right) .
$$

Now the group delay (or transmission time) $T_{g}$ is given by

$$
T_{g}=\frac{1}{2 \pi} \frac{d \phi}{d f}
$$

or

$$
T_{g}=\frac{2 d}{c}\left(1+\frac{1}{2} \frac{h^{2}}{d^{2}}\right)
$$

Evidently, according to this particular method of calculation, which is only applicable at high frequencies, the phase and group delays are equal. Thus the changes in phase delay and propagation time are also equal. However, for low frequencies this single ray treatment is incorrect since all the rays contributing to the received signal must be included.

Waveguide mode theory applied to propagation over long distances gives a phase delay $\phi^{\prime}$, for the dominant mode, of

$$
\phi^{\prime}=\frac{4 \pi d}{\lambda} \sqrt{1-\left(\frac{\lambda}{2 h}\right)^{2}}
$$

which, from (1) can be written as

$$
T_{\phi}^{\prime}=\frac{2 d}{c} \sqrt{1-\left(\frac{\lambda}{2 h}\right)^{2}} .
$$

The group delay or transmission time $T_{\mathrm{g}}^{\prime}$ is, from (4) and (6),

$$
T_{g}^{\prime}=\frac{2 d}{c} / \sqrt{1-\left(\frac{\lambda}{2 h}\right)^{2}}
$$


In this case it is clear that $T_{\phi}^{\prime}$ and $T_{g}^{\prime}$ are no longer equal, and a variation in $h$ produces opposite variations in $T_{\phi}^{\prime}$ and $T_{g}^{\prime}$. For example, at sunrise, or during a solar flare, the height of the ionosphere decreases and thus the phase lag also decreases. However, the transmission time (or group delay) increases as shown by (8), whereas the single ray model would predict a decrease as shown by (5).

Equations (3) and (5) suggest that the origin of the confusion between phase delay and transmission time in VLF propagation over long distances is due to the use of the nondispersive single-ray propagation model. There is, however, overwhelming evidence [Wait, 1962] that long distance VLF propagation can be adequately described in terms of waveguide theory, which even in the simplest (lossless) case shows that the group and phase delays are different. Thus it must be erroneous to equate changes in phase delay and group transmission time.

There are two main practical objections to the description of changes in phase delay as changes in propagation or transmission time. The first of these has been discussed above and is due to the fact that during sunrise, sunset, or solar flares, etc., the phase delays and time delays are of equal magnitude, but change in opposite directions if the waveguide mode theory is used. This is perhaps a trivial objection from the practical point of view, since the only difference is a change of sign. The other objection, however, may be much more important. It is well known [Pierce, 1957] that observations of the relative phase of a VLF signal received over a long path show random variations which, in the case of $16 \mathrm{kc} / \mathrm{s}$ propagation across the North Atlantic, amount to about 0.05 cycles at night. The corresponding $\Delta T_{\phi} \sim 3 \mu$ sec. If these fluctuations are expressed in the latter form, the uninitiated might be led to believe that $\Delta T_{\phi}$ represents the true propagation time variations due to the medium. However, Watt et al. [1961] have pointed out that it is (4) which governs the transmission time. Thus if the phase fluctuations at adjacent frequencies are uncorrelated, the variations in transmission time are likely to be much larger than the observed phase delay variations at a single frequency. In view of this, it seems that the interpretation of phase changes as transmission time changes should be avoided.

To sum up, it has been shown that, for VLF signals, the interpretation of phase changes as changes in transmission time may follow from an over-simplified ray model of propagation. This interpretation gives changes in transmission time which are of the wrong sign for systematic ionosphere height changes and which may be very much in error for random ionospheric changes.

These difficulties can be avoided if it is remembered that dispersion occurs even in a lossless earthionosphere waveguide, and as a result the phase and group delays are different. The temptation to express phase changes as time changes by (1) should either be avoided or, if not, "phase delay," expressed in units of time $\left(T_{\phi}\right)$, and "group transmission time" $\left(T_{g}\right)$ should always be carefully defined and labeled.

\section{References}

Blackband, W. T. (1964), Diurnal changes of transmission time in the arctic propagation of VLF waves, Radio Sci. J. Res. NBS/USNC-URSI 68D, No. 2, 205-210.

Brillouin, L. (1960), Wave propagation and group velocity (Academic Press, New York, N.Y.).

Pierce, J. A. (1957), Intercontinental frequency comparison by VLF radio transmission, Proc. IRE 45, No. 6, 794-803.

Wait, J. R. (1962), Electromagnetic waves in stratified media, ch. IX (Pergamon Press, Ltd., Oxford).

Watt, A. D., R. W. Plush, W. W. Brown, and A. H. Morgan (1961), Worldwide VLF standard frequency and time signal broadcasting, J. Res. NBS 65D (Radio Prop.). No. 6, 617-627.

(Paper 68D11-424) 